\title{
The invariant subspace problem for absolutely $p$-summing operators in Krein spaces
}

\section{Gerald Wanjala*}

\section{"Correspondence:}

wanjalag@yahoo.com; gwanjala@squ.edu.om Department of Mathematics and Statistics, Sultan Qaboos University, P.O. Box 36 PC 123, Al-Khod, Sultanate of Oman

\begin{abstract}
Let $1 \leq p<\infty$, and let $T$ be a bounded linear operator defined on a Krein space $\mathcal{K}$. We prove the existence of a non-positive subspace $\mathcal{L}$ - of $\mathcal{K}$ invariant under $T$ with the assumption that $T$ is absolutely $p$-summing with some further conditions imposed on it.

MSC: Primary 47B50; secondary 46C20; 47B10

Keywords: Krein spaces; invariant subspace; absolutely $p$-summing operator
\end{abstract}

\section{Introduction}

Let $1 \leq p<\infty$. In this article we consider the question of the existence of a non-negative subspace invariant under an absolutely $p$-summing operator $T$ defined on a Krein space $\mathcal{K}$. Recall that a complex linear space $\mathcal{K}$ (or more precisely, $\left(\mathcal{K},\langle\cdot, \cdot\rangle_{\mathcal{K}}\right)$ ) on which a Hermitian form $\langle\cdot, \cdot\rangle_{\mathcal{K}}$ is defined, that is, a complex valued function $\langle\cdot, \cdot\rangle_{\mathcal{K}}$ defined on $\mathcal{K} \times \mathcal{K}$ such that

(i) $\langle x, y\rangle_{\mathcal{K}}=\overline{\langle y, x\rangle}_{\mathcal{K}}$,

(ii) $\langle a x+b y, z\rangle_{\mathcal{K}}=a\langle x, z\rangle_{\mathcal{K}}+b\langle y, z\rangle_{\mathcal{K}}$ for any $x, y, z \in \mathcal{K}$ and $a, b \in \mathbb{C}$,

is called a Krein space if in $\mathcal{K}$ there are two linear manifolds $\mathcal{K}_{+}$and $\mathcal{K}_{-}$such that

$$
\mathcal{K}=\mathcal{K}_{+} \oplus \mathcal{K}_{-},
$$

$\left(\mathcal{K}_{+},\langle\cdot, \cdot\rangle_{\mathcal{K}}\right)$ and $\left(\mathcal{K}_{-},-\langle\cdot, \cdot\rangle_{\mathcal{K}}\right)$ are Hilbert spaces and $\left\langle\mathcal{K}_{+}, \mathcal{K}_{-}\right\rangle_{\mathcal{K}}=\{0\}$. By a linear manifold here we mean a set $\mathcal{M} \subset \mathcal{K}$ with the property that for any two vectors $v, w \in \mathcal{M}$ and any complex numbers $\lambda$ and $\mu$, we have $\lambda v+\mu w \in \mathcal{M}$. It is always assumed that $\mathcal{K}_{+}, \mathcal{K}_{-} \neq\{0\}$. Otherwise, $\left(\mathcal{K},\langle\cdot, \cdot\rangle_{\mathcal{K}}\right)$ or $\left(\mathcal{K},-\langle\cdot, \cdot\rangle_{\mathcal{K}}\right)$ is a Hilbert space. The Hermitian form $\langle\cdot, \cdot\rangle_{\mathcal{K}}$ is called the indefinite inner product of the Krein space $\mathcal{K}$. If, in particular, $\kappa=\min \left(\operatorname{dim} \mathcal{K}_{+}, \operatorname{dim} \mathcal{K}_{-}\right)<\infty$, then $\mathcal{K}$ is called a $\pi_{\kappa}$-space or a Pontryagin space of in$\operatorname{dex} \kappa$. In most of the literature, it is always assumed that $\kappa=\operatorname{dim} \mathcal{K}_{-}$for a $\pi_{\kappa}$-space.

The representation (1.1) is called a fundamental decomposition of the Krein space $\mathcal{K}$ and is not unique in general. Using this decomposition, a Hilbert space inner product $[\cdot, \cdot]_{\mathcal{K}}$ can be defined on $\mathcal{K}$ as follows:

$$
[x, y]_{\mathcal{K}}=\left\langle x_{+}, y_{+}\right\rangle_{\mathcal{K}}-\left\langle x_{-}, y_{-}\right\rangle_{\mathcal{K}},
$$

where $x=x_{+}+x_{-}, y=y_{+}+y_{-}$, with $x_{ \pm}, y_{ \pm} \in \mathcal{K}_{ \pm}$. 
Although decomposition (1.1) is not unique in general, its components $\mathcal{K}_{ \pm}$are uniquely determined and the Hilbert norms generated by different decompositions (1.1) according to (1.2) are equivalent and therefore define the same norm topology. All topological notions such as continuity and convergence in a Krein space are understood to be with respect to this norm topology.

If in the Hilbert space $\left(\mathcal{K},[\cdot, \cdot]_{\mathcal{K}}\right)$, the orthogonal projections onto $\mathcal{K}_{+}$and $\mathcal{K}_{-}$are denoted by $P_{+}$and $P_{-}$respectively, then the operator

$$
J=P_{+}-P_{-}
$$

is called the fundamental symmetry associated with decomposition (1.1) and has the following properties:

(i)

$$
\langle f, g\rangle_{\mathcal{K}}=[J f, g]_{\mathcal{K}}
$$

for any vectors $f$ and $g$ in $\mathcal{K}$, and

(ii) $J^{2}=J, J=J^{*}$, where $J^{*}$ denotes the Krein space adjoint of $J$.

On the other hand, given a Hilbert space $\left(\mathcal{K},[\cdot, \cdot]_{\mathcal{K}}\right)$ and an operator $J$ with the above two properties defined on it (or, more generally, an operator $G$ with $G=G^{*}$ and $0 \in \rho(G)$, the resolvent set of $G$ ), then an indefinite inner product is defined on $\mathcal{K}$ by (1.4) (or, respectively, by the relation

$$
\left.\langle f, g\rangle_{\mathcal{K}}=[G f, g]_{\mathcal{K}}, \quad f, g \in \mathcal{K}\right)
$$

and $\left(\mathcal{K},\langle\cdot, \cdot\rangle_{\mathcal{K}}\right)$ is a Krein space. Because of this construction, Krein spaces are sometimes called $J$-spaces.

The indefinite inner product $\langle\cdot, \cdot\rangle_{\mathcal{K}}$ on a Krein space $\mathcal{K}$ gives rise to a classification of elements of $\mathcal{K}$. An element $k \in \mathcal{K}$ is called positive, negative, or neutral if $\langle k, k\rangle_{\mathcal{K}}>0$, $\langle k, k\rangle_{\mathcal{K}}<0$, or $\langle k, k\rangle_{\mathcal{K}}=0$ respectively. A linear manifold or a subspace $\mathcal{L}$ in $\mathcal{K}$ is called indefinite if it contains both positive and negative elements. We say that $\mathcal{L}$ is semi-definite if it is not indefinite. A semi-definite subspace $\mathcal{L}$ is called non-negative (positive, uniformly positive) if $\langle x, x\rangle \geq 0(\langle x, x\rangle>0,\langle x, x\rangle \geq \delta\|x\|,(\delta>0))$ for all $x$ in $\mathcal{L}$. A non-positive (negative, uniformly negative) subspace is defined in a similar way. We say that the subspace $\mathcal{L}$ is definite if $\langle x, x\rangle=0$ if and only if $x=0$.

If a non-negative subspace $\mathcal{L}$ admits no nontrivial non-negative extensions, then it is called a maximal non-negative subspace. Maximal non-positive (positive, negative, etc.) subspaces in $\mathcal{K}$ are similarly defined.

Before winding up this review on Krein spaces, we note that the Cauchy-Schwarz inequality,

$$
\left|\langle x, y\rangle_{\mathcal{K}}\right| \leq\|x\|\|y\|, \quad x, y \in \mathcal{K}
$$

holds in a Krein space setting. This can be deduced from the following:

$$
\begin{aligned}
\left|\langle x, y\rangle_{\mathcal{K}}\right|^{2} & =\left|\left[x_{+}, y_{+}\right]_{\mathcal{K}}-\left[x_{-}, y_{-}\right]_{\mathcal{K}}\right|^{2} \\
& \leq\left(\left\|x_{+}\right\|\left\|y_{+}\right\|+\left\|x_{-}\right\|\left\|y_{-}\right\|\right)^{2}
\end{aligned}
$$




$$
\begin{aligned}
& \leq\left(\left\|x_{+}\right\|^{2}+\left\|x_{-}\right\|^{2}\right)\left(\left\|y_{+}\right\|^{2}+\left\|y_{-}\right\|^{2}\right) \\
& =\|x\|^{2}\|y\|^{2} .
\end{aligned}
$$

More details on Krein space theory can be found in [1-4], and [5].

We now turn to the main problem under consideration here, which is the question of the existence of semi-definite invariant subspaces for absolutely $p$-summing operators on a Krein space $\mathcal{K}$. For various classes of operators, this problem has been a subject of research since the early days of the theory of operators in spaces with an indefinite metric. One of the first results in this direction was obtained by Pontryagin [6] in 1944 for self-adjoint operators acting on $\pi_{\kappa}$-spaces. In particular, he proved the following theorem.

Theorem 1.1 Let $T$ be a self adjoint operator in a $\pi_{\kappa}$ space. Then there exists a maximal non-negative T-invariant subspace $\mathcal{M}$ (of dimension $\kappa$ ) such that the spectrum of the restriction $\left.T\right|_{\mathcal{M}}$ lies in the closed upper half plane.

One year earlier, Sobolev [7] had solved a similar problem for the case $\kappa=1$. After Pontryagin's result, the problem on the existence of invariant maximal semi-definite subspaces turned out to be the focus of attention in the theory of operators in Pontryagin and Krein spaces. In this regard, we note the articles by Krein [8, 9], Langer [10-12], Azizov [13, 14], and some others. Krein [8] obtained an analogue of Pontryagin's theorem for unitary operators on $\pi_{\kappa}$ spaces and developed a new approach to the invariant subspace problem in spaces with an indefinite metric. An important generalization of Pontryagin's result was obtained by Krein [9] and Langer [10]. As this subject developed, theorems on the existence of $T$-invariant subspaces were obtained for other classes of operators. Langer [11,12] proved the existence of maximal definite invariant subspaces for a wider class of operators, the definitizable operators. Krein and Langer [15], and independently Azizov [14], showed that Pontryagin's theorem remains true for maximal dissipative operators. Further details on the development of this problem till the 1990s can be found in $[2,16]$. More recently, this problem has been considered in a series of papers by Shkalikov [17-21] and also by Azizov and Gridneva [22], Azizov and Khatskevich [23], and Pyatkov [24, 25].

Let $1 \leq p<\infty$. It is the aim of this paper to prove the existence of a non-positive invariant subspace for an absolutely $p$-summing operator $T$ (defined below) acting on a Krein space $\mathcal{K}$ and having the following properties:

(i) there exists a circle $\Gamma:=\{\xi \in \mathbb{C}:|\xi|=r\}$ which separates the spectrum of $T$ for which the scalar multiple $\xi R(\xi)$ of the resolvent operator

$$
R(\xi):=(T-\xi I)^{-1} \quad(\xi \in \Gamma)
$$

is expansive, that is,

$$
|\xi|^{2}\langle R(\xi) x, R(\xi) x\rangle_{\mathcal{K}} \geq\langle x, x\rangle_{\mathcal{K}}
$$

for all $x \in \mathcal{K}$;

(ii) there exists some real number $\alpha<0$ such that

$$
[R(\xi) x, R(\xi) x]_{\mathcal{K}} \leq \xi[R(\xi) x, \alpha x]_{\mathcal{K}}+\bar{\xi}[\alpha x, R(\xi) x]_{\mathcal{K}} .
$$


The subset of $p$-absolutely summing operators having both properties stated above is nonempty as we shall show at the end of this article.

\section{Preliminaries}

We begin this section by defining the class of absolutely $p$-summing operators whose theory was developed in the late sixties mainly by Pietsch [26]. The literature on these operators is very extensive and varied. References [27-29], and [30] are probably among the most extensive ones in this regard.

The first results marking the beginning of the theory of these operators are contained in Grothendieck's paper [31], where one of the classical theorems of the time (Grothendieck's theorem) was obtained. It says that every bounded linear operator from $\ell_{1}$ to $\ell_{2}$ is absolutely summing (see the definition below).

Let $\mathcal{H}_{1}$ and $\mathcal{H}_{2}$ be Hilbert spaces with inner products $[\cdot, \cdot]_{\mathcal{H}_{1}}$ and $[\cdot, \cdot]_{\mathcal{H}_{2}}$ respectively, and let $1 \leq p<\infty$. A bounded linear operator $T: \mathcal{H}_{1} \rightarrow \mathcal{H}_{2}$ is called absolutely $p$-summing if there exists a constant $c>0$ such that for each positive integer $m$ and any vectors $h_{1}, h_{2}, \ldots, h_{m}$ in $\mathcal{H}_{1}$, we have

$$
\left(\sum_{i=1}^{m}\left\|T h_{i}\right\|^{p}\right)^{1 / p} \leq c \cdot \sup \left\{\left(\sum_{i=1}^{m}\left|\left[h_{i}, h\right]_{\mathcal{H}_{1}}\right|^{p}\right)^{1 / p}: h \in \mathcal{H}_{1},\|h\| \leq 1\right\},
$$

or equivalently,

$$
\left(\sum_{i=1}^{m}\left\|T h_{i}\right\|^{p}\right)^{1 / p} \leq c \cdot \sup \left\{\left(\sum_{i=1}^{m}\left|h^{*}\left(h_{i}\right)\right|^{p}\right)^{1 / p}: h^{*} \in \mathcal{H}_{1}^{*},\left\|h^{*}\right\| \leq 1\right\},
$$

where $\mathcal{H}_{1}^{*}$ is the topological dual of $\mathcal{H}_{1}$. The smallest constant $c$ for which (2.1) holds is denoted by $\pi_{p}(T)$, while $\Pi_{p}\left(\mathcal{H}_{1}, \mathcal{H}_{2}\right)$ stands for the set of all absolutely $p$-summing operators from $\mathcal{H}_{1}$ into $\mathcal{H}_{2}$. If $\mathcal{H}_{1}=\mathcal{H}_{2}=\mathcal{H}$, then this set is denoted by $\Pi_{p}(\mathcal{H})$. For the case $p=1$, these operators are simply referred to as absolutely summing operators. Using the Minkowski inequality for sums, one can easily prove that $\Pi_{p}\left(\mathcal{H}_{1}, \mathcal{H}_{2}\right)$ is a linear subspace of $B\left(\mathcal{H}_{1}, \mathcal{H}_{2}\right)$, the set of all bounded linear operators from $\mathcal{H}_{1}$ into $\mathcal{H}_{2}$.

Closely related to the class of absolutely $p$-summing operators is the class of absolutely $(p, q)$-summing operators for $1 \leq p, q<\infty$. If inequality (2.1) is replaced by

$$
\left(\sum_{i=1}^{m}\left\|T h_{i}\right\|^{p}\right)^{1 / p} \leq c \cdot \sup \left\{\left(\sum_{i=1}^{m}\left|\left[h_{i}, h\right]_{\mathcal{H}_{1}}\right|^{q}\right)^{1 / q}: h \in \mathcal{H}_{1},\|h\| \leq 1\right\},
$$

then the operator $T$ is called absolutely $(p, q)$-summing. In this case, the smallest constant $c$ for which (2.2) holds is denoted by $\pi_{p, q}(T)$, while $\Pi_{p, q}\left(\mathcal{H}_{1}, \mathcal{H}_{2}\right)$ denotes the class of absolutely $(p, q)$-summing operators from $\mathcal{H}_{1}$ into $\mathcal{H}_{2}$. The class $\Pi_{p, q}\left(\mathcal{H}_{1}, \mathcal{H}_{2}\right)$ can also be characterized as a collection of operators $T: \mathcal{H}_{1} \rightarrow \mathcal{H}_{2}$ which take weakly $q$-summable sequences in $\mathcal{H}_{1}$ to strongly $p$-summable sequences in $\mathcal{H}_{2}$. We note that if $p<q$, then $\Pi_{p, q}\left(\mathcal{H}_{1}, \mathcal{H}_{2}\right)=\{0\}$. As before, we write $\Pi_{p, q}(\mathcal{H})$ for $\Pi_{p, q}(\mathcal{H}, \mathcal{H})$.

We now look at the above definitions in the context of Krein spaces. Let $\mathcal{K}_{1}$ and $\mathcal{K}_{2}$ be Krein spaces with inner products $\langle\cdot, \cdot\rangle_{\mathcal{K}_{1}}$ and $\langle\cdot, \cdot\rangle_{\mathcal{K}_{2}}$ respectively, and let $T: \mathcal{K}_{1} \rightarrow \mathcal{K}_{2}$ be 
a bounded linear operator. If we replace (2.1) and (2.2) with

$$
\left(\sum_{i=1}^{m}\left\|T k_{i}\right\|^{p}\right)^{1 / p} \leq c \cdot \sup \left\{\left(\sum_{i=1}^{m}\left|\left\langle k_{i}, k\right\rangle_{\mathcal{K}_{1}}\right|^{p}\right)^{1 / p}: k \in \mathcal{K}_{1},\|k\| \leq 1\right\} \text {, }
$$

and

$$
\left(\sum_{i=1}^{m}\left\|T k_{i}\right\|^{p}\right)^{1 / p} \leq c \cdot \sup \left\{\left(\sum_{i=1}^{m}\left|\left\langle k_{i}, k\right\rangle_{\mathcal{K}_{1}}\right|^{q}\right)^{1 / q}: k \in \mathcal{K}_{1},\|k\| \leq 1\right\},
$$

respectively, we get the definitions of absolutely $p$-summing and absolutely $(p, q)$-summing operators between Krein spaces $\mathcal{K}_{1}$ and $\mathcal{K}_{2}$. The rest of the notation remains as in the Hilbert space case.

Below is an example of an absolutely $p$-summing operator on an arbitrary Krein space $\mathcal{K}$ for $1 \leq p<\infty$.

Example 2.1 Let $\mathcal{K}$ be a Krein space with the indefinite inner product $\langle\cdot, \cdot\rangle_{\mathcal{K}}$, and let $h$ and $k$ be fixed elements of $\mathcal{K}$ with $\|h\| \leq 1$. Let $1 \leq p<\infty$ and define an operator $T: \mathcal{K} \rightarrow \mathcal{K}$ by

$$
T x=\langle x, h\rangle_{\mathcal{K}} k .
$$

Then $T$ is clearly well defined and linear. The fact that this operator is bounded and absolutely $p$-summing with $\pi_{p}(T) \leq\|k\|$ follows from

$$
\|T x\|=\|\langle x, h\rangle k\|=\left|\langle x, h\rangle_{\mathcal{K}}\right|\|k\|
$$

since

$$
\|T x\|^{p} \leq\|k\|^{p}\left|\langle x, h\rangle_{\mathcal{K}}\right|^{p}
$$

and so

$$
\sum_{i=1}^{m}\left\|T x_{i}\right\|^{p} \leq\|k\|^{p} \sum_{i=1}^{m}\left|\left\langle x_{i}, h\right\rangle_{\mathcal{K}}\right|^{p}
$$

for each positive integer $m$ and any choice of vectors $x_{1}, x_{2}, \ldots, x_{m}$ in $\mathcal{K}$.

Equipped with this example, we can construct many others by simply taking a linear combination of operators of the form $(2.5)$ since $\Pi_{p}(\mathcal{K})$ is a linear subspace of $B(\mathcal{K})$.

Consider a Krein space $\mathcal{K}$ with the indefinite inner product $\langle\cdot, \cdot\rangle_{\mathcal{K}}$. By $|\mathcal{K}|$ we denote the Hilbert space associated with the Krein space $\mathcal{K}$, that is, the space $\mathcal{K}$ together with the positive definite inner product $[\cdot, \cdot]_{\mathcal{K}}=\langle J \cdot, \cdot\rangle_{\mathcal{K}}$, where $J$ is the operator defined in (1.3).

Remark 2.2 Let $\mathcal{K}_{1}$ and $\mathcal{K}_{2}$ be Krein spaces, and let $T: \mathcal{K}_{1} \rightarrow \mathcal{K}_{2}$ be a bounded linear operator. Then $T$ is absolutely $p$-summing if and only if it is absolutely $p$-summing as an operator from $\left|\mathcal{K}_{1}\right|$ into $\left|\mathcal{K}_{2}\right|$. 
Proof Let $1 \leq p<\infty$ and suppose that $T: \mathcal{K}_{1} \rightarrow \mathcal{K}_{2}$ is absolutely $p$-summing. Since $\|J k\|=$ $\|k\|$ and $J$ maps the open unit ball onto itself in a one-to-one way, then for some constant $c>0$ and any positive integer $m$, we have

$$
\begin{aligned}
\left(\sum_{i=1}^{m}\left\|T k_{i}\right\|^{p}\right)^{1 / p} & \leq c \cdot \sup \left\{\left(\sum_{i=1}^{m}\left|\left\langle k_{i}, k\right\rangle_{\mathcal{K}_{1}}\right|^{p}\right)^{1 / p}: k \in \mathcal{K}_{1},\|k\| \leq 1\right\} \\
& =c \cdot \sup \left\{\left(\sum_{i=1}^{m}\left|\left[k_{i}, J k\right]_{\left|\mathcal{K}_{1}\right|}\right|^{p}\right)^{1 / p}: k \in \mathcal{K}_{1},\|k\| \leq 1\right\} \\
& =c \cdot \sup \left\{\left(\sum_{i=1}^{m}\left|\left[k_{i}, J k\right]_{\left|\mathcal{K}_{1}\right|}\right|^{p}\right)^{1 / p}: J k \in\left|\mathcal{K}_{1}\right|,\|J k\| \leq 1\right\} \\
& =c \cdot \sup \left\{\left(\sum_{i=1}^{m}\left|\left[k_{i}, \tilde{k}\right]_{\left|\mathcal{K}_{1}\right|}\right|^{p}\right)^{1 / p}: \tilde{k} \in\left|\mathcal{K}_{1}\right|,\|\tilde{k}\| \leq 1\right\},
\end{aligned}
$$

where we have set $J k=\tilde{k}$.

The reverse implication can be proved in a similar way and is omitted.

We conclude this section by introducing the concept of a Riesz projector (see [32] for more properties of this operator). Let $\Gamma$ be a Cauchy contour, and let $g: \Gamma \rightarrow \mathcal{X}$ be a continuous function on $\Gamma$ with values in a complete normed linear space $\mathcal{X}$. Then (as in complex function theory) the integral

$$
\frac{1}{2 \pi i} \int_{\Gamma} g(\lambda) d \lambda
$$

is defined as a Stielties integral, where the corresponding Stieltjes sum converges in the norm of $\mathcal{X}$. Thus, the value of (2.6) is a vector in $\mathcal{X}$ which appears as a limit (in the norm of $\mathcal{X}$ ) of the corresponding Stieltjes sum. From this definition, it is clear that

$$
F\left(\frac{1}{2 \pi i} \int_{\Gamma} g(\lambda) d \lambda\right)=\frac{1}{2 \pi i} \int_{\Gamma} F(g(\lambda)) d \lambda
$$

for any continuous linear functional $F$ on $\mathcal{X}$. Note that the integrand of the second integral in (2.7) is just a scalar valued function.

Of interest to us is the case when $\mathcal{X}$ is the Banach space $B\left(X_{1}, X_{2}\right)$ consisting of all bounded linear operators from the Banach space $X_{1}$ into the Banach space $X_{2}$. So, let $X_{1}$ and $X_{2}$ be Banach spaces, and let $g: \Gamma \rightarrow B\left(X_{1}, X_{2}\right)$ be a continuous function. Then the value of the integral (2.6) is a bounded linear operator from $X_{1}$ into $X_{2}$, and for each $x \in X_{1}$, we have

$$
\left(\frac{1}{2 \pi i} \int_{\Gamma} g(\lambda) d \lambda\right) x=\frac{1}{2 \pi i} \int_{\Gamma} g(\lambda) x d \lambda .
$$

Let $T$ be a bounded linear operator on an arbitrary Hilbert space $\mathcal{H}$. Suppose that $T$ has a separated spectrum, and let $\sigma_{T}$ be an isolated part of the spectrum of $T$. Let $\Gamma$ be a Jordan closed rectifiable contour lying in $\rho(T)$, the resolvent set of $T$, and containing $\sigma_{T}$. 
Suppose further that $\sigma(T) \backslash \sigma_{T}$ lies outside this contour. Then the value of the integral

$$
P=-\frac{1}{2 \pi i} \oint_{\Gamma}(T-\lambda I) d \lambda,
$$

which is understood to be the strong limit of the integral sums, is a bounded linear operator on $\mathcal{H}$ and is called a Riesz projector. The following theorem, which can be found in [2] enumerates some of the various properties of this integral.

\section{Theorem 2.3}

(a) The operator P does not depend on the choice of the contour $\Gamma$ isolating the set $\sigma_{T}$ and it is a projection;

(b) The subspaces $P \mathcal{H}$ and $(I-P) \mathcal{H}$ are invariant under $T$ and $\sigma\left(\left.T\right|_{P \mathcal{H}}\right)=\sigma_{T}$, $\sigma\left(\left.T\right|_{(I-P) \mathcal{H}}\right)=\sigma(T) \backslash \sigma_{T}$.

(c) If $T$ is a bounded linear operator and $\sigma_{T}=\sigma(T)$, then $P=I$.

In this theorem, it is understood that $T$ is an everywhere defined operator on $\mathcal{H}$. Otherwise, the Riesz projector $P$ has the additional property that $P \mathcal{H} \subset \mathcal{D}(T)$, the domain of $T$ in $\mathcal{H}$.

\section{Non-positive invariant subspace}

Let $p$ be such that $1 \leq p<\infty$. In this section we show the existence of a non-positive invariant subspace for an absolutely $p$-summing operators $T$ acting on a Krein space $\mathcal{K}$ satisfying the two conditions stated at the end of Section 1.

Let $1 \leq p<\infty$, and let $\mathcal{K}$ be a Krein space. The following lemma establishes a contractivelike relation for any $T \in \pi_{p}(\mathcal{K})$ with the constant $\pi_{p}(T) \leq 1$.

Lemma 3.1 Let $\mathcal{K}$ be a Krein space with some fixed fundamental decomposition, and for $1 \leq p<\infty$, let $T \in \Pi_{p}(\mathcal{K})$ be such that $\pi_{p}(T) \leq 1$. Then with respect to this fixed fundamental decomposition, the inequality

$$
\langle T x, T x\rangle_{\mathcal{K}} \leq[x, x]_{\mathcal{K}}
$$

holds.

Proof The Cauchy-Schwarz inequality together with the fact that $\pi_{p}(T) \leq 1$ yields the following series of inequalities:

$$
\begin{aligned}
\langle T x, T x\rangle & \leq|\langle T x, T x\rangle| \\
& \leq\|T x\|\|T x\| \leq \pi_{p}(T)^{2} \sup _{\|y\| \leq 1}|\langle x, y\rangle|^{2} \\
& \leq \sup _{\|y\| \leq 1}|\langle x, y\rangle|^{2} \\
& \leq\|x\|^{2}=[x, x]_{\mathcal{K}} .
\end{aligned}
$$

Let $\mathcal{K}$ be a Krein space. For $1 \leq p<\infty$, let $T \in \Pi_{p}(\mathcal{K})$. In the theorem below, which is our main result, we prove the existence of a non-positive invariant subspace for $T$ under the 
assumption that the operator $\xi(T-\xi I)^{-1}$ is contractive, where $\xi \in \Gamma$ for some specified contour $\Gamma$.

Theorem 3.2 Let $\mathcal{K}$ be a Krein space, and for $1 \leq p<\infty$, let $T \in \Pi_{p}(\mathcal{K})$. Assume that $T$ has the following properties:

(i) there exists a positive real number $r$ for which the circle $\Gamma:=\{\xi:|\xi|=r\}$ separates the spectrum of $T$ and is such that the scalar multiple $\xi R(\xi)$ of the resolvent operator

$$
R(\xi):=(T-\xi I)^{-1} \quad(\xi \in \Gamma)
$$

is expansive, that is, $|\xi|^{2}\langle R(\xi) x, R(\xi) x\rangle_{\mathcal{K}} \geq\langle x, x\rangle_{\mathcal{K}}$,

(ii) there exists some real number $\alpha<0$ such that

$$
[R(\xi) x, R(\xi) x]_{\mathcal{K}} \leq \xi[R(\xi) x, \alpha x]_{\mathcal{K}}+\bar{\xi}[\alpha x, R(\xi) x]_{\mathcal{K}}
$$

Then there exists a non-positive subspace $\mathcal{L}_{-} \subset \mathcal{K}$ which is invariant under $T$.

Proof For $1 \leq p<\infty$, let $T \in \Pi_{p}(\mathcal{K})$ and assume that $T$ satisfies the conditions of the theorem. For any real number $a$ with $0<a \leq 1$, the operator $a T$ belongs to $\Pi_{p}(\mathcal{K})$ and satisfies the conditions of the theorem with $\xi$ replaced by $a \xi$. It is clear that the first two conditions are satisfied. To see that the third condition is satisfied, we first recall that $R(a \xi):=(a T-a \xi I)^{-1}=\frac{1}{a}(T-\xi I)^{-1}=\frac{1}{a} R(\xi)$. Then

$$
\begin{aligned}
{[R(a \xi) x, R(a \xi) x]_{\mathcal{K}} } & =\frac{1}{a^{2}}[R(\xi) x, R(\xi) x]_{\mathcal{K}} \\
& \leq \frac{\xi}{a^{2}}[R(\xi) x, \alpha x]_{\mathcal{K}}+\frac{\bar{\xi}}{a^{2}}[\alpha x, R(\xi) x]_{\mathcal{K}} \\
& =\frac{\xi}{a}[R(a \xi) x, \alpha x]_{\mathcal{K}}+\frac{\bar{\xi}}{a}[\alpha x, R(a \xi) x]_{\mathcal{K}} \\
& =a \xi[R(a \xi) x, \beta x]_{\mathcal{K}}+a \xi[\beta x, R(a \xi) x]_{\mathcal{K}},
\end{aligned}
$$

where $\beta=\frac{\alpha}{a^{2}}$. Hence, the third condition holds.

Since the operators $T$ and $a T$ have the same invariant subspaces, we will consider the case when the constant $\pi_{p}(T) \leq 1$. So, without loss of generality, assume that the operator $T$ is such that $\pi_{p}(T) \leq 1$. Lemma 3.1 implies that for $k \in \mathcal{K}$ with $k \neq 0$ and $\xi \in \Gamma$, we have

$$
\left\langle T(T-\xi I)^{-1} k, T(T-\xi I)^{-1} k\right\rangle_{\mathcal{K}} \leq\left[(T-\xi I)^{-1} k,(T-\xi I)^{-1} k\right]_{\mathcal{K}} .
$$

Let $(T-\xi I)^{-1} k=z$. Then $k=(T-\xi I) z=T z-\xi z$ and so $T z=k+\xi z$. This implies that

$$
\begin{aligned}
\langle T z, T z\rangle_{\mathcal{K}} & =\langle k+\xi z, k+\xi z\rangle_{\mathcal{K}} \\
& =\langle k, k\rangle_{\mathcal{K}}+\xi\langle z, k\rangle_{\mathcal{K}}+\bar{\xi}\langle k, z\rangle_{\mathcal{K}}+|\xi|^{2}\langle z, z\rangle_{\mathcal{K}} .
\end{aligned}
$$


From inequality (3.1) we see that

$$
\begin{aligned}
\langle k, k\rangle_{\mathcal{K}}+\xi\left\langle(T-\xi I)^{-1} k, k\right\rangle_{\mathcal{K}}+\bar{\xi}\left\langle k,(T-\xi I)^{-1} k\right\rangle_{\mathcal{K}} \\
+|\xi|^{2}\left\langle(T-\xi I)^{-1} k,(T-\xi I)^{-1} k\right\rangle_{\mathcal{K}} \\
\leq\left[(T-\xi I)^{-1} k,(T-\xi I)^{-1} k\right]_{\mathcal{K}} .
\end{aligned}
$$

Since

$$
|\xi|^{2}\left\langle(T-\xi I)^{-1} k,(T-\xi I)^{-1} k\right\rangle_{\mathcal{K}} \geq\langle k, k\rangle_{\mathcal{K}}
$$

we see that

$$
\begin{aligned}
& \langle k, k\rangle_{\mathcal{K}}+\xi\left\langle(T-\xi I)^{-1} k, k\right\rangle_{\mathcal{K}}+\bar{\xi}\left\langle k,(T-\xi I)^{-1} k\right\rangle_{\mathcal{K}}+\langle k, k\rangle_{\mathcal{K}} \\
& \quad \leq\left[(T-\xi I)^{-1} k,(T-\xi I)^{-1} k\right]_{\mathcal{K}}
\end{aligned}
$$

Since by assumption

$$
\left[(T-\xi I)^{-1} k,(T-\xi I)^{-1} k\right]_{\mathcal{K}} \leq \xi\left[(T-\xi I)^{-1} k, \alpha k\right]_{\mathcal{K}}+\bar{\xi}\left[\alpha k,(T-\xi I)^{-1} k\right]_{\mathcal{K}}
$$

for some real number $\alpha$, inequality (3.2) can be written as

$$
\begin{aligned}
& \langle k, k\rangle_{\mathcal{K}}+\xi\left\langle(T-\xi I)^{-1} k, k\right\rangle_{\mathcal{K}}+\bar{\xi}\left\langle k,(T-\xi I)^{-1} k\right\rangle_{\mathcal{K}}+\langle k, k\rangle_{\mathcal{K}} \\
& \quad \leq \xi\left[(T-\xi I)^{-1} k, \alpha k\right]_{\mathcal{K}}+\xi\left[\alpha k,(T-\xi I)^{-1} k\right]_{\mathcal{K}}
\end{aligned}
$$

which is equivalent to

$$
\begin{aligned}
& \langle k, k\rangle_{\mathcal{K}}+\xi\left\langle(T-\xi I)^{-1} k, k\right\rangle_{\mathcal{K}}+\bar{\xi}\left\langle k,(T-\xi I)^{-1} k\right\rangle_{\mathcal{K}}+\langle k, k\rangle_{\mathcal{K}} \\
& \leq \xi\left\langle(T-\xi I)^{-1} k, \alpha J k\right\rangle_{\mathcal{K}}+\bar{\xi}\left\langle\alpha J k,(T-\xi I)^{-1} k\right\rangle_{\mathcal{K}}
\end{aligned}
$$

We now introduce the Riesz projector

$$
P=-\frac{1}{2 \pi i} \int_{|\xi|=r}(T-\xi I)^{-1} d \xi .
$$

First, we put $\xi=r e^{i \theta}$ in (3.3), divide either side of this equation by $2 \pi$, and integrate from 0 to $2 \pi$ with respect to $\theta$ to get

$$
\begin{array}{r}
2\langle k, k\rangle_{\mathcal{K}}+\frac{1}{2 \pi} \int_{0}^{2 \pi} r e^{i \theta}\left\langle\left(T-r e^{i \theta} I\right)^{-1} k, k\right\rangle_{\mathcal{K}} d \theta+\frac{1}{2 \pi} \overline{\int_{0}^{2 \pi} r e^{i \theta}\left\langle\left(T-r e^{i \theta} I\right)^{-1} k, k\right\rangle_{\mathcal{K}} d \theta} \\
\leq \frac{1}{2 \pi} \int_{0}^{2 \pi} r e^{i \theta}\left\langle\left(T-r e^{i \theta} I\right)^{-1} k, \alpha J k\right\rangle_{\mathcal{K}} d \theta+\frac{1}{2 \pi} \overline{\int_{0}^{2 \pi} r e^{i \theta}\left\langle\left(T-r e^{i \theta} I\right)^{-1} k, \alpha J k\right\rangle_{\mathcal{K}} d \theta}
\end{array}
$$


Hence,

$$
\begin{aligned}
2\langle k, k\rangle_{\mathcal{K}}+\frac{1}{2 \pi i} \int_{0}^{2 \pi} i r e^{i \theta}\left\langle\left(T-r e^{i \theta} I\right)^{-1} k, k\right\rangle_{\mathcal{K}} d \theta \\
+\frac{\frac{1}{2 \pi i} \int_{0}^{2 \pi} i r e i^{i}\left\langle\left(T-r e^{i \theta} I\right)^{-1} k, k\right\rangle_{\mathcal{K}} d \theta}{\leq} \\
\frac{\frac{1}{2 \pi i} \int_{0}^{2 \pi} i r e^{i \theta}\left\langle\left(T-r e^{i \theta} I\right)^{-1} k, \alpha J k\right\rangle_{\mathcal{K}} d \theta}{+\frac{1}{2 \pi i} \int_{0}^{2 \pi} i r e^{i \theta}\left\langle\left(T-r e^{i \theta} I\right)^{-1} k, \alpha J k\right\rangle_{\mathcal{K}} d \theta}
\end{aligned}
$$

which we write as

$$
\begin{array}{r}
2\langle k, k\rangle_{\mathcal{K}}+\frac{1}{2 \pi i} \int_{|\xi|=r}\left\langle(T-\xi I)^{-1} k, k\right\rangle_{\mathcal{K}} d \xi+\overline{\frac{1}{2 \pi i} \int_{|\xi|=r}\left\langle(T-\xi I)^{-1} k, k\right\rangle_{\mathcal{K}} d \xi} \\
\leq \frac{1}{2 \pi i} \int_{\mid \xi=r}\left\langle(T-\xi I)^{-1} k, \alpha J k\right\rangle_{\mathcal{K}} d \xi+\overline{\frac{1}{2 \pi i} \int_{|\xi|=1}\left\langle\left(T-r e^{i \theta} I\right)^{-1} k, \alpha J k\right\rangle_{\mathcal{K}} d \xi}
\end{array}
$$

We now use the fact that $\langle\cdot, k\rangle$ and $\langle\cdot, \alpha J k\rangle$ are bounded linear functionals on $\mathcal{K}$ together with (2.7) to rewrite the above inequality as

$$
2\langle k, k\rangle_{\mathcal{K}}-\langle P k, k\rangle_{\mathcal{K}}-\langle k, P k\rangle_{\mathcal{K}} \leq-\langle P k, \alpha J k\rangle_{\mathcal{K}}-\langle\alpha J k, P k\rangle_{\mathcal{K}}
$$

where

$$
P=-\frac{1}{2 \pi i} \int_{|\xi|=r}(T-\xi I)^{-1} d \xi .
$$

Denote by $\mathcal{L}_{-}$the subspace $(I-P) \mathcal{K}$. Inequality (3.4) implies that for $k \in \mathcal{L}_{-}$, we have $\langle k, k\rangle_{\mathcal{K}} \leq 0$, and Theorem 2.3 ensures that this is the required invariant subspace. This concludes the proof.

Note that the requirement $\alpha<0$ in the theorem cannot be dropped. If we do, then (3.4) yields the invalid inequality

$$
2\langle k, J k\rangle_{\mathcal{K}} \leq 0
$$

for all $k \in P \mathcal{K}$.

We conclude this article by showing that an operator with the properties stated in Theorem 3.2 above does exist, and therefore, our result does not hold on an empty set.

Example 3.3 Let $\mathcal{K}$ be a Krein space and fix a negative element $h \in \mathcal{K}$ with the property that $\|h\|<1$, and there exists a circle $\Gamma$ centered at the origin and small enough such that $2(\operatorname{Re} \xi)>\langle h, h\rangle_{\mathcal{K}}$ and $\left|\langle h, h\rangle_{\mathcal{K}}-\xi\right|^{2} \geq\|h\|^{2}$ for all $\xi \in \Gamma$. Such an element $h$ exists (for example, we can pick a suitable $h$ in $\mathcal{K}_{-}$). Define a bounded linear operator $T$ on $\mathcal{K}$ by

$$
T x=\langle x, h\rangle_{\mathcal{K}} h .
$$


This operator satisfies all the conditions of Theorem 3.2 and clearly has a non-positive invariant subspace. To show that this is the case, we first note that from Example 2.1, one readily sees that $T$ is absolutely $p$-summing with $\pi(T)<1$ for $1 \leq p<\infty$. The only eigenvalues of this operator are $\lambda_{1}=0$ and $\lambda_{2}=\langle h, h\rangle_{\mathcal{K}}$. Since $T$ has finite rank, these eigenvalues are also the only spectral values of $T$. Hence, the circle $\Gamma$ lies in the resolvent set $\rho(T)$ of $T$ and separates its spectrum. For any $\xi \in \Gamma$, the resolvent operator $R_{\xi}(T):=$ $(T-\xi I)^{-1}$ is defined. A little manipulation shows that $R_{\xi}(T)$ is given by

$$
R_{\xi}(T)=\frac{1}{\xi}\left[\frac{\langle\cdot, h\rangle_{\mathcal{K}} h}{\langle h, h\rangle_{\mathcal{K}}-\xi}-I\right] .
$$

Now

$$
\begin{aligned}
\left\langle R_{\xi}(T) x, R_{\xi}(T) x\right\rangle_{\mathcal{K}}= & \frac{1}{|\xi|^{2}}\left\{\left|\frac{\langle x, h\rangle_{\mathcal{K}}}{\langle h, h\rangle_{\mathcal{K}}-\xi}\right|^{2}\langle h, h\rangle_{\mathcal{K}}-\frac{\left|\langle h, x\rangle_{\mathcal{K}}\right|^{2}}{\langle h, h\rangle_{\mathcal{K}}-\bar{\xi}}\right. \\
& \left.-\frac{\left|\langle h, x\rangle_{\mathcal{K}}\right|^{2}}{\langle h, h\rangle_{\mathcal{K}}-\xi}+\langle x, x\rangle_{\mathcal{K}}\right\} \\
= & \frac{1}{|\xi|^{2}}\left\{\left|\frac{\langle x, h\rangle_{\mathcal{K}}}{\langle h, h\rangle_{\mathcal{K}}-\xi}\right|^{2}\langle h, h\rangle_{\mathcal{K}}\right. \\
& \left.-\frac{\left|\langle h, x\rangle_{\mathcal{K}}\right|^{2}\left(\langle h, h\rangle_{\mathcal{K}}-\xi\right)+\left|\langle h, x\rangle_{\mathcal{K}}\right|^{2}\left(\langle h, h\rangle_{\mathcal{K}}-\bar{\xi}\right)}{\left|\langle h, h\rangle_{\mathcal{K}}-\xi\right|^{2}}+\langle x, x\rangle_{\mathcal{K}}\right\} \\
= & \frac{1}{|\xi|^{2}}\left\{\frac{\left|\langle h, x\rangle_{\mathcal{K}}\right|^{2}(\xi+\bar{\xi})}{\left.\left|\langle h, h\rangle_{\mathcal{K}}-\xi\right|^{2}-\left|\frac{\langle x, h\rangle_{\mathcal{K}}}{\langle h, h\rangle_{\mathcal{K}}-\xi}\right|^{2}\langle h, h\rangle_{\mathcal{K}}+\langle x, x\rangle_{\mathcal{K}}\right\}}\right. \\
= & \frac{1}{|\xi|^{2}}\left\{\left|\frac{\langle x, h\rangle_{\mathcal{K}}}{\langle h, h\rangle_{\mathcal{K}}-\xi}\right|^{2}\left[2(\operatorname{Re} \xi)-\langle h, h\rangle_{\mathcal{K}}\right]+\langle x, x\rangle_{\mathcal{K}}\right\} \\
> & \frac{1}{|\xi|^{2}}\langle x, x\rangle_{\mathcal{K} .}
\end{aligned}
$$

The last inequality holds because $2(\operatorname{Re} \xi)>\langle h, h\rangle_{\mathcal{K}}$. Hence,

$$
|\xi|^{2}\left\langle R_{\xi}(T) x, R_{\xi}(T) x\right\rangle_{\mathcal{K}}>\langle x, x\rangle_{\mathcal{K}}
$$

and so the operator $\xi R_{\xi}(T)$ is expansive.

We now show that there exists some real number $\alpha<0$ such that

$$
[R(\xi) x, R(\xi) x]_{\mathcal{K}} \leq \xi[R(\xi) x, \alpha x]_{\mathcal{K}}+\bar{\xi}[\alpha x, R(\xi) x]_{\mathcal{K}}
$$

Now,

$$
\begin{aligned}
{[R(\xi) x, R(\xi) x]_{\mathcal{K}}=} & \frac{1}{|\xi|^{2}}\left[\frac{\langle x, h\rangle_{\mathcal{K}} h}{\langle h, h\rangle_{\mathcal{K}}-\xi}-x, \frac{\langle x, h\rangle_{\mathcal{K}} h}{\langle h, h\rangle_{\mathcal{K}}-\xi}-x\right]_{\mathcal{K}} \\
= & \frac{1}{|\xi|^{2}}\left\{\frac{\left|\langle x, h\rangle_{\mathcal{K}}\right|^{2}}{\left|\langle h, h\rangle_{\mathcal{K}}-\xi\right|^{2}}[h, h]_{\mathcal{K}}-\frac{\langle h, x\rangle_{\mathcal{K}}[x, h]_{\mathcal{K}}}{\langle h, h\rangle_{\mathcal{K}}-\bar{\xi}}\right. \\
& \left.-\frac{\langle x, h\rangle_{\mathcal{K}}[h, x]_{\mathcal{K}}}{\langle h, h\rangle_{\mathcal{K}}-\xi}+[x, x]_{\mathcal{K}}\right\} \\
\leq & \frac{1}{|\xi|^{2}}\left\{\frac{[x, x]_{\mathcal{K}}\|h\|^{4}}{\left|\langle h, h\rangle_{\mathcal{K}}-\xi\right|^{2}}-\frac{\langle h, x\rangle_{\mathcal{K}}[x, h]_{\mathcal{K}}}{\langle h, h\rangle_{\mathcal{K}}-\bar{\xi}}-\frac{\langle x, h\rangle_{\mathcal{K}}[h, x]_{\mathcal{K}}}{\langle h, h\rangle_{\mathcal{K}}-\xi}+[x, x]_{\mathcal{K}}\right\}
\end{aligned}
$$




$$
\begin{aligned}
& \leq \frac{1}{|\xi|^{2}}\left\{[x, x]_{\mathcal{K}}-\frac{\langle h, x\rangle_{\mathcal{K}}[x, h]_{\mathcal{K}}}{\langle h, h\rangle_{\mathcal{K}}-\bar{\xi}}-\frac{\langle x, h\rangle_{\mathcal{K}}[h, x]_{\mathcal{K}}}{\langle h, h\rangle_{\mathcal{K}}-\xi}+[x, x]_{\mathcal{K}}\right\} \\
& =\frac{1}{|\xi|^{2}}\left\{2[x, x]_{\mathcal{K}}-\frac{\langle x, h\rangle_{\mathcal{K}}[h, x]_{\mathcal{K}}}{\langle h, h\rangle-\xi}-\frac{\langle h, x\rangle_{\mathcal{K}}[x, h]_{\mathcal{K}}}{\langle h, h\rangle-\bar{\xi}}\right\}
\end{aligned}
$$

where we have used the Cauchy-Schwarz inequality to obtain the first inequality and the fact that $\left|\langle h, h\rangle_{\mathcal{K}}-\xi\right|^{2} \geq\|h\|^{2}$ and $\|h\|<1$ to obtain the second inequality.

We also have that

$$
\begin{aligned}
\xi\left[R(\xi) x,-\frac{1}{|\xi|^{2}} x\right]_{\mathcal{K}}+\bar{\xi}\left[-\frac{1}{|\xi|^{2}} x, R(\xi) x\right]_{\mathcal{K}} \\
=\left[\frac{\langle x, h\rangle_{\mathcal{K}} h}{\langle h, h\rangle-\xi}-x,-\frac{1}{|\xi|^{2}} x\right]_{\mathcal{K}} \\
\quad+\left[-\frac{1}{|\xi|^{2}} x, \frac{\langle x, h\rangle_{\mathcal{K}} h}{\langle h, h\rangle-\xi}-x\right]_{\mathcal{K}} \\
=-\frac{1}{|\xi|^{2}} \frac{\langle x, h\rangle_{\mathcal{K}}[h, x]_{\mathcal{K}}}{\langle h, h\rangle-\xi}+\frac{1}{|\xi|^{2}}[x, x]_{\mathcal{K}}-\frac{1}{|\xi|^{2}} \frac{\langle h, x\rangle_{\mathcal{K}}[x, h]_{\mathcal{K}}}{\langle h, h\rangle-\bar{\xi}}+\frac{1}{|\xi|^{2}}[x, x]_{\mathcal{K}} \\
=\frac{1}{|\xi|^{2}}\left\{2[x, x]_{\mathcal{K}}-\frac{\langle x, h\rangle_{\mathcal{K}}[h, x]_{\mathcal{K}}}{\langle h, h\rangle-\xi}-\frac{\langle h, x\rangle_{\mathcal{K}}[x, h]_{\mathcal{K}}}{\langle h, h\rangle-\bar{\xi}}\right\} .
\end{aligned}
$$

With $\alpha=-\frac{1}{|\xi|^{2}}$, we see that

$$
[R(\xi) x, R(\xi) x]_{\mathcal{K}} \leq \xi[R(\xi) x, \alpha x]_{\mathcal{K}}+\bar{\xi}[\alpha x, R(\xi) x]_{\mathcal{K}}
$$

\section{Competing interests}

The author declares that he has no competing interests.

\section{Received: 3 July 2012 Accepted: 19 October 2012 Published: 31 October 2012}

\section{References}

1. Ando, T: Linear Operators on Krein Spaces. Hokkaido University, Research Institute of Applied Electricity, Division of Applied Mathematics, Sapporo (1979)

2. Azizov, TY, lokhvidov, El: Linear operators in spaces with an indefinite metric. In: Itogi Nauki i Techniki, Matem. Analis, vol. 17. Akad. Nauk. SSSR, Moscow (1979)

3. Bognar, J: Indefinite Inner Product Spaces. Springer, New York (1974)

4. Dritschel, MA, Rovnyak, J: Extension Theorems for Contraction Operators in Krein Spaces. Operator Theory: Adv. Appl, vol. 47, pp. 221-305. Birkhäuser, Basel (1990)

5. Iohvidov, IS, Krein, MG, Langer, H: Introduction to the Spectral Theory of Operators in Spaces with an Indefinite Metric. Akademie Verlag, Berlin (1982)

6. Pontrysgin, LS: Hermitian operators in spaces with an indefinite metric. IzV. Akad. Nauk SSSR, Ser. Mat. 8, 243-280 (1944) (in Russian)

7. Sobolev, LS: Motion of a symmetric top with a cavity filled with liquid. Zh. Matem. Tekhn. Fiz 3, 20-55 (1960)

8. Krein, MG: On an application of the fixed point principle in the theory of operators in a space with indefinite metric. Usp. Mat. Nauk 50, 180-190 (1950) (in Russian)

9. Krein, MG: A new application of fixed point principle in the theory of operators is a space with indefinite metric. Dokl. Akad. Nauk SSSR 154(5), 1023-1026 (1964) (in Russian)

10. Langer, H: On J-Hermitian operators. Dokl. Akad. Nauk SSSR 134(2), 263-266 (1962) (in Russian)

11. Langer, $\mathrm{H}$ : Invariant subspaces for a class of a class of operators in spaces with indefinite metric. J. Funct. Anal. 19 232-241 (1975)

12. Langer, H: Spectral Functions of Definitizable Operators in Krein Spaces. Lect. Notes in Math., vol. 948 (1982)

13. Azizov, TY: Invariant subspaces and criteria for the completeness of the system of root vectors of J-dissipative operators in the Pontrjagin space. Sov. Math. Dokl. 200(5), 1513-1516 (1971)

14. Azizov, TY: Dissipative operators in Hilbert space with indefinite metric. Izv. Akad. Nauk SSSR, Ser. Mat. 37(3), 639-662 (1973) (in Russian). English translation in Math. USSR, Izv. 7 (1973)

15. Krein, MG, Langer, $\mathrm{H}$ : On definite subspaces and generalized resolvents of Hermitian operators in spaces $\Pi_{\kappa}$. Funkc. Anal. Prilozh. 5(3), 54-69 (1971) (in Russian). English translation in Funct. Anal. Appl. 5 (1971) 
16. Azizov, TY, lokhvidov, El: Foundations of Theory of Linear Operators in Spaces with an Indefinite Metric. Nauka, Moscow (1986) (in Russian)

17. Shkalikov, AA: On the existence of invariant subspaces of dissipative operators in a space with an indefinite metric. Fundam. Prikl. Mat. 5(2), 627-635 (1999)

18. Shkalikov, AA: On invariant subspaces of dissipative operators in a space with indefinite metric arXiv.math/0412116v1 [math.FA] (2004)

19. Shkalikov, AA: On the invariant subspace problem for dissipative operators in Krein spaces. arXiv.math/0512465v2 [math.FA] (2005)

20. Shkalikov, AA: Invariant subspaces of dissipative operators in a space with an indefinite metric. Tr. Mat. Inst. Steklova 248, 294-303 (2005)

21. Shkalikov, AA: Dissipative operators in a Krein space. Invariant subspaces and properties of restrictions. Funkc. Anal. Prilozh. 41(2), 93-110 (2007)

22. Azizov, TY, Gridneva, IV: On invariant subspaces of J-dissipative operators. Ukr. Math. Bull. 6(1), 1-13 (2009)

23. Azizov, TY, Khatskevich, VA: A Theorem on Existence of Invariant Subspaces for J-binoncontractive Operators. Operator Theory: Adv. Appl., vol. 198, pp. 41-48. Birkhäuser, Basel (2009)

24. Pyatkov, SG: Maximal semidefinite invariant subspaces for J-dissipative operators. In: Spectral Theory, Mathematica System Theory, Evolution Equations, Differential and Difference Equations. Oper. Theory: Adv. Appl., vol. 221, pp. 549-570 (2012)

25. Pyatkov, SG: On the existence of maximal semidefinite invariant subspaces for J-dissipative operators. Sb. Math. 230(2), 234-256 (2012)

26. Pietsch, A: Absolut p-summierende Abbildungen in normeierten Raumen. Stud. Math. 28, 333-353 (1968)

27. Diestel, J, Jarchow, H, Tonge, A: Absolutely Summing Operators. Cambridge University Press, Cambridge (1995)

28. Lindenstraus, J, Pelczynski, A: Absolutely summing operators in $\boldsymbol{\mathcal { L }}_{p}$ spaces and their applications. Stud. Math. 29, 275-326 (1968)

29. Makarov, BM: $p$-absolutely summing operators and some of their applications. St. Petersburg Math. J. 3(2), 227-298 (1992)

30. Pisier, G: Factorization of Linear Operators and the Geometry of Banach Spaces. CBMS, vol. 60. Amer. Math. Soc., Providence (1986)

31. Grothendieck, A: Resumé de la théorie métrique des produits tensoriels topologigues. Bol. Soc. Mat. São Paulo 8, 1-79 (1956)

32. Gohberg, I, Goldberg, S, Kaashoek, M: Classes of Linear Operators I. Birkhäuser, Basel (1990)

doi:10.1186/1029-242X-2012-254

Cite this article as: Wanjala: The invariant subspace problem for absolutely $p$-summing operators in Krein spaces.

Journal of Inequalities and Applications 2012 2012:254.

\section{Submit your manuscript to a SpringerOpen ${ }^{\odot}$ journal and benefit from:}

- Convenient online submission

- Rigorous peer review

- Immediate publication on acceptance

Open access: articles freely available online

- High visibility within the field

- Retaining the copyright to your article 